\title{
Factors affecting approach selection for minimally invasive versus open surgery in the treatment of adult spinal deformity: analysis of a prospective, nonrandomized multicenter study
}

\author{
Paul Park, MD, ${ }^{1}$ Khoi D. Than, MD, ${ }^{2}$ Praveen V. Mummaneni, MD, ${ }^{3}$ Pierce D. Nunley, MD, ${ }^{4}$ \\ Robert K. Eastlack, MD, ${ }^{5}$ Juan S. Uribe, MD, ${ }^{6}$ Michael Y. Wang, MD, ${ }^{7}$ Vivian Le, BS, ${ }^{3}$ \\ Richard G. Fessler, MD, PhD, ${ }^{8}$ David O. Okonkwo, MD, PhD, ${ }^{9}$ Adam S. Kanter, MD, ${ }^{9}$ \\ Neel Anand, MD, ${ }^{10}$ Dean Chou, MD, ${ }^{3}$ Kai-Ming G. Fu, MD, PhD, ${ }^{11}$ Alexander F. Haddad, BS, ${ }^{12}$ \\ Christopher I. Shaffrey, MD, ${ }^{13}$ Gregory M. Mundis Jr., MD, ${ }^{5}$ and the International Spine \\ Study Group
}

\begin{abstract}
${ }^{1}$ Department of Neurosurgery, University of Michigan, Ann Arbor, Michigan; ${ }^{2}$ Department of Neurological Surgery, Oregon Health \& Science University, Portland, Oregon; ${ }^{2}$ Department of Neurosurgery, University of California, San Francisco, California; ${ }^{4}$ Spine Institute of Louisiana, Shreveport, Louisiana; ${ }^{5}$ Department of Orthopaedic Surgery, Scripps Clinic, La Jolla, California; ${ }^{6}$ Department of Neurosurgery, Barrow Neurological Institute, Phoenix, Arizona; ${ }^{7}$ Department of Neurosurgery, University

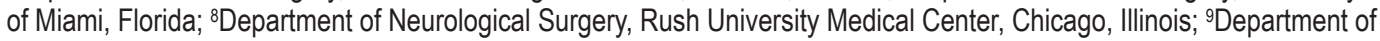
Neurosurgery, University of Pittsburgh Medical Center, Pittsburgh, Pennsylvania; ${ }^{10}$ Department of Orthopaedics, Cedars-Sinai Medical Center, Los Angeles, California; ${ }^{11}$ Department of Neurosurgery, Cornell Medical Center, New York, New York; ${ }^{12}$ School of Medicine, University of California, San Francisco, California; and ${ }^{13}$ Departments of Orthopaedic Surgery and Neurosurgery, Duke University, Durham, North Carolina
\end{abstract}

OBJECTIVE Surgical decision-making and planning is a key factor in optimizing outcomes in adult spinal deformity (ASD). Minimally invasive spinal (MIS) strategies for ASD have been increasingly used as an option to decrease postoperative morbidity. This study analyzes factors involved in the selection of either a traditional open approach or a minimally invasive approach to treat ASD in a prospective, nonrandomized multicenter trial. All centers had at least 5 years of experience in minimally invasive techniques for ASD.

METHODS The study enrolled 268 patients, of whom 120 underwent open surgery and 148 underwent MIS surgery. Inclusion criteria included age $\geq 18$ years, and at least one of the following criteria: coronal curve (CC) $\geq 20^{\circ}$, sagittal vertical axis $(\mathrm{SVA})>5 \mathrm{~cm}$, pelvic tilt $(\mathrm{PT})>25^{\circ}$, or thoracic kyphosis $(\mathrm{TK})>60^{\circ}$. Surgical approach selection was made at the discretion of the operating surgeon. Preoperative significant differences were included in a multivariate logistic regression analysis to determine odds ratios (ORs) for approach selection.

RESULTS Significant preoperative differences $(p<0.05)$ between open and MIS groups were noted for age $(61.9$ vs 66.7 years), numerical rating scale (NRS) back pain score $(7.8$ vs 7$), \mathrm{CC}\left(36^{\circ}\right.$ vs $\left.26.1^{\circ}\right), \mathrm{PT}\left(26.4^{\circ}\right.$ vs $\left.23^{\circ}\right), \mathrm{T}^{1}$ pelvic angle (TPA; $25.8^{\circ}$ vs $21.7^{\circ}$ ), and pelvic incidence-lumbar lordosis (PI-LL; $19.6^{\circ}$ vs $\left.14.9^{\circ}\right)$. No significant differences in BMI (29 vs $28.5 \mathrm{~kg} / \mathrm{m}^{2}$ ), NRS leg pain score (5.2 vs 5.7$)$, Oswestry Disability Index (48.4 vs 47.2), Scoliosis Research Society 22-item questionnaire score (2.7 vs 2.8$)$, PI ( $58.3^{\circ}$ vs $\left.57.1^{\circ}\right)$, LL ( $38.9^{\circ}$ vs $\left.42.3^{\circ}\right)$, or SVA (73.8 $\mathrm{mm}$ vs $\left.60.3 \mathrm{~mm}\right)$ were found. Multivariate analysis found that age (OR 1.05, $p=0.002)$, VAS back pain score (OR 1.21, $p=0.016), C C$ (OR 1.03, $p<0.001)$, decompression (OR 4.35, $p<0.001$ ), and TPA (OR 1.09, $p=0.023$ ) were significant factors in approach selection.

CONCLUSIONS Increasing age was the primary driver for selecting MIS surgery. Conversely, increasingly severe deformities and the need for open decompression were the main factors influencing the selection of traditional open surgery. As experience with MIS surgery continues to accumulate, future longitudinal evaluation will reveal if more expe-

ABBREVIATIONS ALIF = anterior lumbar interbody fusion; ASD = adult spinal deformity; CC = coronal curve; LL = lumbar lordosis; LLIF = lateral lumbar interbody fusion; MIS = minimally invasive spinal; NRS = numerical rating scale; ODI = Oswestry Disability Index; OLIF = oblique lumbar interbody fusion; OR = odds ratio; PI = pelvic incidence; PT = pelvic tilt; SRS-22 = Scoliosis Research Society 22-item questionnaire; SVA = sagittal vertical axis; TK = thoracic kyphosis; TLIF = transforaminal lumbar interbody fusion; TPA = T1 pelvic angle.

SUBMITTED February 7, 2020. ACCEPTED April 16, 2020.

INCLUDE WHEN CITING Published online June 19, 2020; DOI: 10.3171/2020.4.SPINE20169. 
rience, use of specialized treatment algorithms, refinement of techniques, and technology will expand surgeon adoption of MIS techniques for adult spinal deformity.

https://thejns.org/doi/abs/10.3171/2020.4.SPINE20169

KEYWORDS adult spinal deformity; interbody fusion; minimally invasive surgery; spinal surgery; surgical approach

$\mathrm{M}$ INIMALLY invasive spinal (MIS) approaches for adult spinal deformity (ASD) have been shown to be effective alternatives to traditional surgery in appropriately selected patients. ${ }^{1-3}$ The major benefit of MIS surgery reflects a decreased exposure-related morbidity resulting in less blood loss and potentially quicker recovery. ${ }^{4}$ MIS approaches to deformity correction typically involve use of interbody fusion, most commonly lateral lumbar interbody fusion (LLIF) and oblique lumbar interbody fusion (OLIF), as well as tubular transforaminal lumbar interbody fusion (TLIF) and mini-open anterior lumbar interbody fusion (ALIF). These various interbody fusion techniques are combined with percutaneous instrumentation to achieve deformity correction. The evolution from traditional open surgery to the adoption of circumferential MIS techniques to treat ASD is often challenging. The learning curve and the adoption timeline may be many years. The major concern that persists among surgeons is that MIS deformity surgery, particularly without use of advanced techniques such as anterior column realignment, has limited the ability to create significant lordosis. ${ }^{5}$

This study retrospectively analyzed data from a prospective, nonrandomized multicenter trial in which surgeons had at least 5 years of experience with MIS techniques for ASD. Our goal was to identify the factors that influenced the participating surgeons in their selection of either a traditional open approach or an MIS approach.

\section{Methods}

IRB approval was obtained at each of the 10 participating centers (see Appendix). Only surgeons who performed both MIS and traditional open deformity surgery participated in the trial. Enrolled patients provided informed consent to be involved in this observational investigation. Inclusion criteria included age $\geq 18$ years, and at least one of the following criteria: coronal curve (CC) $\geq 20^{\circ}$, sagittal vertical axis (SVA) $>5 \mathrm{~cm}$, pelvic tilt $(\mathrm{PT})>25^{\circ}$, or thoracic kyphosis $(\mathrm{TK})>60^{\circ}$. Surgical approach selection was at the discretion of the operating surgeon.

\section{Radiological Assessment}

All patients had preoperative anteroposterior and lateral scoliosis radiographs. Standard radiographic parameters were assessed, including CC, SVA, T1 pelvic angle (TPA), lumbar lordosis (LL), pelvic incidence (PI), PT, and PI-LL mismatch. The TPA is an alternative method to assess global sagittal alignment that accounts for pelvic compensation and is an angle defined by a line drawn from the center of $\mathrm{T} 1$ to the bifemoral heads and a line drawn from the bifemoral heads to the mid-S1 endplate. ${ }^{6}$ Unlike SVA, it is an angular measurement, so calibration is not needed. All imaging was reviewed at a single site
(Hospital for Special Surgery, New York, New York) for uniformity, and calculations were performed using specialized software (SpineView, ENSAM ParisTech).

\section{Clinical Assessment}

Several surveys were utilized to assess outcomes, including the numerical rating scale (NRS) for back and leg pain, Oswestry Disability Index (ODI), and Scoliosis Research Society 22-item questionnaire (SRS-22). The NRS ranges from 0 (no pain) to 10 (unbearable pain). The ODI ranges from 0 to 100 , with increasing values associated with worsening disability. The SRS-22 assesses 5 domains (function, pain, self-image, mental health, and satisfaction) and ranges from a mean score of $1-5$, with higher scores reflecting better outcomes.

\section{Surgical Technique}

Participating surgeons assessed each enrolled patient and determined the surgical approach (open vs MIS) based on the presentation, comorbidities, physical examination, and radiographic imaging. There was no specific surgical protocol for the group, and the surgeon was allowed to select the approach based on what he or she considered the best option to achieve optimal outcomes. Minimally invasive approaches used a combination of LLIF/OLIF, ALIF, and/or TLIF, with percutaneous instrumentation. Open approaches involved traditional posterior muscle dissection with placement of pedicle screw fixation in combination with interbody fusion and/or osteotomies.

\section{Statistical Analysis}

Statistical analysis involved t-tests and nonparametric tests (Mann-Whitney U-test) for comparisons between MIS and open surgery patients. Preoperative variables with significant differences found on the univariate analysis were included in a multivariate logistic regression analysis to determine odds ratios (ORs) for approach selection. The variables included were age, baseline back pain, baseline CC, baseline PI-LL, baseline PT, if a decompression was performed, and baseline TPA.

\section{Results}

A total of 268 patients were enrolled in the investigation from June 2015 to January 2018. There were 120 open and 148 MIS-treated patients (Table 1). Significant preoperative differences $(\mathrm{p}<0.05)$ between open and MIS groups were noted with univariate analysis for age (61.9 vs 66.7 years), NRS back pain score (7.8 vs 7), CC $\left(36^{\circ}\right.$ vs $\left.26.1^{\circ}\right)$, PT $\left(26.4^{\circ}\right.$ vs $\left.23^{\circ}\right)$, TPA $\left(25.8^{\circ}\right.$ vs $\left.21.7^{\circ}\right)$, and PI-LL $\left(19.6^{\circ}\right.$ vs $\left.14.9^{\circ}\right)$. No significant differences were found in univariate analysis when comparing the open versus the MIS group in baseline preoperative BMI (29 vs $\left.28.5 \mathrm{~kg} / \mathrm{m}^{2}\right)$, NRS leg 
TABLE 1. Demographics, surgical characteristics, patientreported outcomes, and radiographic imaging of open and MIS surgical approach patients

\begin{tabular}{|c|c|c|c|}
\hline Variable & Open Surgery & MIS Surgery & $p$ Value \\
\hline No. of patients & 120 & 148 & \\
\hline Age, yrs & $61.9(12.6)$ & $66.7(9.7)$ & 0.001 \\
\hline $\mathrm{BMI}, \mathrm{kg} / \mathrm{m}^{2}$ & $29(8.9)$ & $28.5(5.4)$ & 0.707 \\
\hline Decompression & $70(58.3 \%)$ & $49(33.1 \%)$ & $<0.001$ \\
\hline NRS back pain score & $7.8(2.1)$ & $7(2.0)$ & $<0.001$ \\
\hline NRS leg pain score & $5.2(3.4)$ & $5.7(2.93)$ & 0.38 \\
\hline ODI & $48.4(18.1)$ & $47.2(14.5)$ & 0.565 \\
\hline SRS-22 score & $2.7(0.6)$ & $2.8(0.6)$ & 0.23 \\
\hline Sacral slope, ${ }^{\circ}$ & $32.1(11.7)$ & $34.2(12.0)$ & 0.189 \\
\hline $\mathrm{CC},{ }^{\circ}$ & $36(20.8)$ & $26.1(14.8)$ & 0.001 \\
\hline $\mathrm{PT}, \stackrel{\circ}{ }$ & $26.4(10.4)$ & $23(8.7)$ & 0.002 \\
\hline $\mathrm{PI},{ }^{\circ}$ & $58.3(13.9)$ & $57.1(13.4)$ & 0.402 \\
\hline $\mathrm{PI}-\mathrm{LL},{ }^{\circ}$ & $19.6(20.9)$ & $14.9(16.5)$ & 0.034 \\
\hline $\mathrm{LL}^{\circ}{ }^{\circ}$ & $38.9(19.7)$ & $42.3(19.0)$ & 0.176 \\
\hline SVA, mm & $73.8(72.7)$ & $60.3(58.6)$ & 0.191 \\
\hline $\mathrm{TPA}^{\circ}{ }^{\circ}$ & $25.8(13.28)$ & $21.7(10.0)$ & 0.008 \\
\hline
\end{tabular}

Data are presented as mean (SD) unless otherwise indicated. Boldface type indicates statistical significance.

pain score (5.2 vs 5.7), ODI (48.4 vs 47.2), SRS-22 (2.7 vs $2.8)$, PI $\left(58.3^{\circ}\right.$ vs $\left.57.1^{\circ}\right)$, LL $\left(38.9^{\circ}\right.$ vs $\left.42.3^{\circ}\right)$, or SVA $(73.8$ vs $60.3 \mathrm{~mm}$ ).

In the open group, 99 patients (83\%) returned for 1-year follow-up, with a mean of 1.1 years (Fig. 1). As Table 2 shows, the open group showed significant improvement in NRS back (7.8 to 3.73) and leg (5.2 to 3.1) pain scores, ODI (48.4 to 30.1), and SRS-22 (2.7 to 3.5) scores. Radiographic parameters also improved with PT $\left(26.4^{\circ}\right.$ to $\left.23.7^{\circ}\right)$, PI-LL $\left(19.6^{\circ}\right.$ to $\left.5.9^{\circ}\right)$, LL $\left(38.9^{\circ}\right.$ to $\left.54.1^{\circ}\right)$, SVA (73.8 $\mathrm{mm}$ to $36.9 \mathrm{~mm})$, and TPA $\left(25.8^{\circ}\right.$ to $\left.20.0^{\circ}\right)$. In the MIS surgery group, 90 patients $(61 \%)$ presented for 1-year follow-up, with a mean of 1.0 years (Fig. 2). Similar to the open surgery group, there were significant improvements in NRS back (7.0 to 2.9) and leg (5.7 to 2.5) pain scores, ODI (47.2 to 27.3), and SRS-22 scores (2.8 to 3.5; Table $3)$. Radiographic improvements were also noted, including PT $\left(23.0^{\circ}\right.$ to $\left.20.8^{\circ}\right)$, PI-LL $\left(14.9^{\circ}\right.$ to $\left.6.9^{\circ}\right)$, LL $\left(42.3^{\circ}\right.$ to $\left.49.8^{\circ}\right)$, SVA $(60.3$ to $39.8 \mathrm{~mm})$, and TPA $\left(21.7^{\circ}\right.$ to $\left.18.1^{\circ}\right)$.

Multivariate analysis found that age $(\mathrm{OR} 1.05, \mathrm{p}=$ $0.002)$, back pain (OR 1.21, p = 0.016), $\mathrm{CC}(\mathrm{OR} 1.03, \mathrm{p}$ $<0.001$ ), need for decompression (OR 4.35, p < 0.001), and TPA (OR 1.09, $\mathrm{p}=0.023)$ were significant factors in approach selection (Table 4). For every 1-year increase in age there was a 5\% increase in selecting an MIS surgical approach. Conversely, for every 1-point increase in VAS back pain, there was a $21 \%$ increase in choosing an open approach. Similarly, for every $1^{\circ}$ increase in CC and TPA, there was a $3 \%$ and $9 \%$ higher likelihood of selecting an open approach, respectively. The most notable variable was whether a patient required a decompression of the neural elements, which showed a 335\% increased likelihood of selecting an open approach.

\section{Discussion}

Initial studies by Glassman et al. ${ }^{7,8}$ showed a correlation between positive sagittal balance and worse clinical outcomes. Subsequent investigation by Lafage et al. ${ }^{9}$
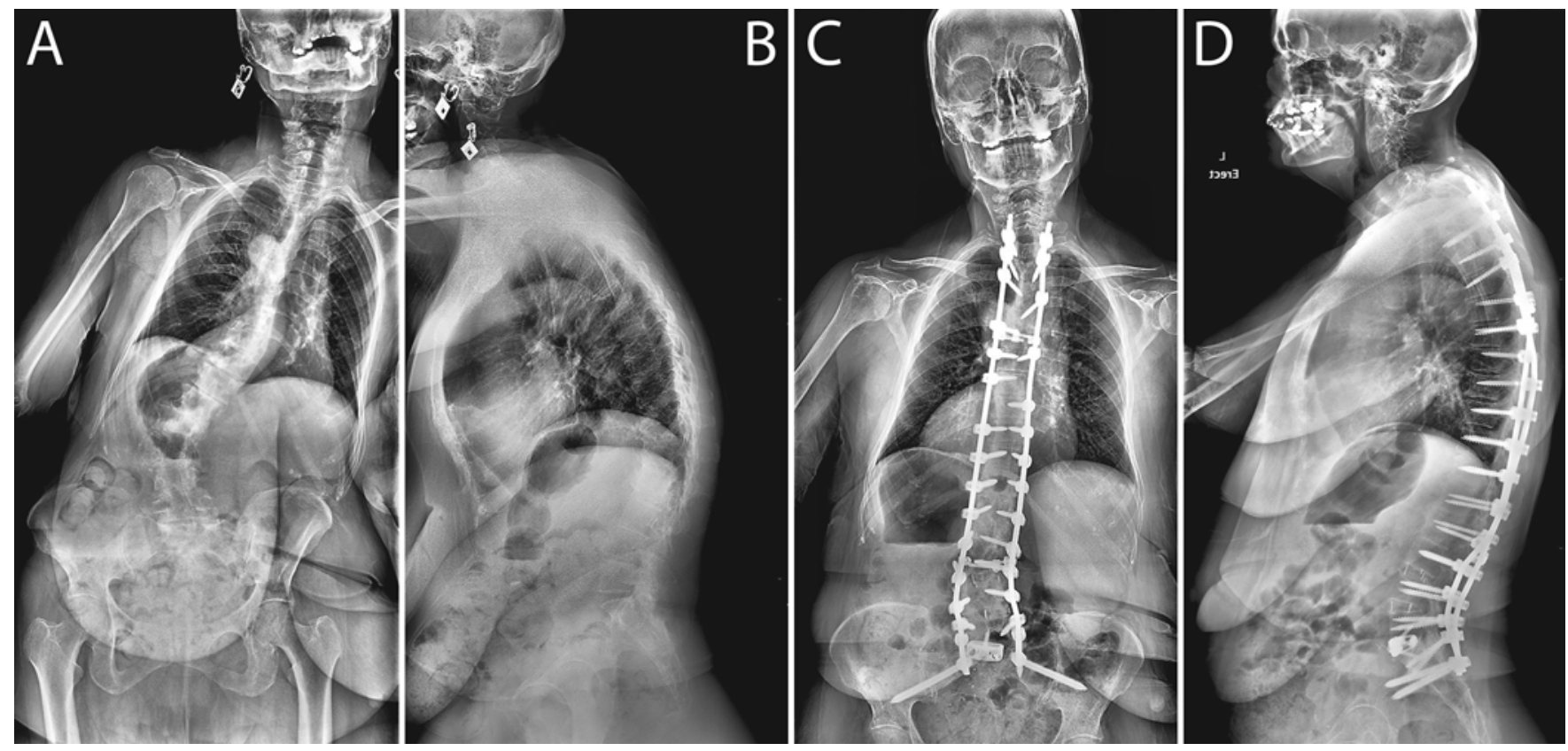

FIG. 1. Preoperative anteroposterior $(\mathbf{A})$ and lateral $(\mathbf{B})$ radiographs and postoperative anteroposterior $(\mathbf{C})$ and lateral (D) radiographs from a 74-year-old woman with kyphoscoliosis who underwent traditional open surgery. Her preoperative values were: SVA

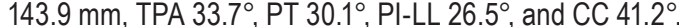


Park et al.

TABLE 2. One-year outcomes in patients who underwent open surgery $(n=99)$

\begin{tabular}{|c|c|c|c|}
\hline Variable & Baseline & 1-Yr Postop & $p$ Value \\
\hline NRS back pain score & $7.8(2.1)$ & $3.73(3.0)$ & $<0.001$ \\
\hline NRS leg pain score & $5.2(3.4)$ & $3.1(3.1)$ & $<0.001$ \\
\hline ODI & $48.4(18.1)$ & $30.1(20.7)$ & $<0.001$ \\
\hline SRS-22 score & $2.7(0.6)$ & $3.5(0.8)$ & $<0.001$ \\
\hline Sacral slope, ${ }^{\circ}$ & $32.1(11.7)$ & $35.3(12.2)$ & 0.005 \\
\hline $\mathrm{CC},{ }^{\circ}$ & $11.9(20.8)$ & $6.42(26.0)$ & $<0.001$ \\
\hline $\mathrm{PT},{ }^{\circ}$ & $26.4(10.4)$ & $23.7(10.9)$ & $<0.001$ \\
\hline $\mathrm{PI}{ }^{\circ}$ & $58.3(13.9)$ & $60.0(14.3)$ & 0.625 \\
\hline $\mathrm{PI}-\mathrm{LL},{ }^{\circ}$ & $19.6(20.9)$ & $5.9(16.4)$ & $<0.001$ \\
\hline $\mathrm{LL}^{\circ}{ }^{\circ}$ & $38.9(19.7)$ & $54.1(16.7)$ & $<0.001$ \\
\hline SVA, mm & $73.8(72.7)$ & $36.9(57.7)$ & $<0.001$ \\
\hline $\mathrm{TPA}^{\circ}$ & $25.8(13.3)$ & $20.0(11.5)$ & $<0.001$ \\
\hline
\end{tabular}

Data are presented as mean (SD).

demonstrated the importance of the pelvis. Schwab et al. ${ }^{10}$ suggested that optimal realignment parameters are SVA $<5 \mathrm{~cm}, \mathrm{PT}<20^{\circ}$, and PI-LL mismatch $\pm 10^{\circ}$. Although age can modify radiographic alignment goals, appropriate realignment by either a traditional open or an MIS surgical approach is considered a key goal to optimize clinical outcomes. Whether an MIS surgical approach can achieve similar correction and outcomes to traditional open surgery for all types of deformity is unclear and has led to the development of treatment guidelines, such as the minimally invasive spinal deformity surgery (MISDEF2) algorithm. ${ }^{11}$ The goal of the MISDEF2 algorithm was to guide surgeons in deciding whether a particular deformity could be treated by MIS surgery. However, MISDEF2 is based only on radiographic alignment criteria and does not factor in other relevant patient data, such as clinical presentation.

This study analyzed the decision-making of surgeons who perform both traditional open and MIS deformity surgery to identify factors impacting approach selection. While spinopelvic malalignment (as reflected in the TPA) and degree of scoliosis were drivers for approach selection, notably, other variables were also influential, including age, back pain, and whether decompression was needed. The lack of SVA being a primary driver may be due to the fact that pelvic compensation is not accounted for with SVA, in contrast to TPA. Given that the higher likelihood was relatively modest, at a $9 \%$ increase per $1^{\circ}$ increase in the TPA, it suggests that only severe spinopelvic malalignment resulted in choosing an open approach. The smaller $3 \%$ increased likelihood per $1^{\circ}$ increase in coronal curvature probably reflects the relative improved ability of MIS approaches to achieve coronal correction. Increasing back pain was also a driver for selecting an open approach. The cause of this finding is not obvious but may be related to the perception by the surgeon that a maximal correction
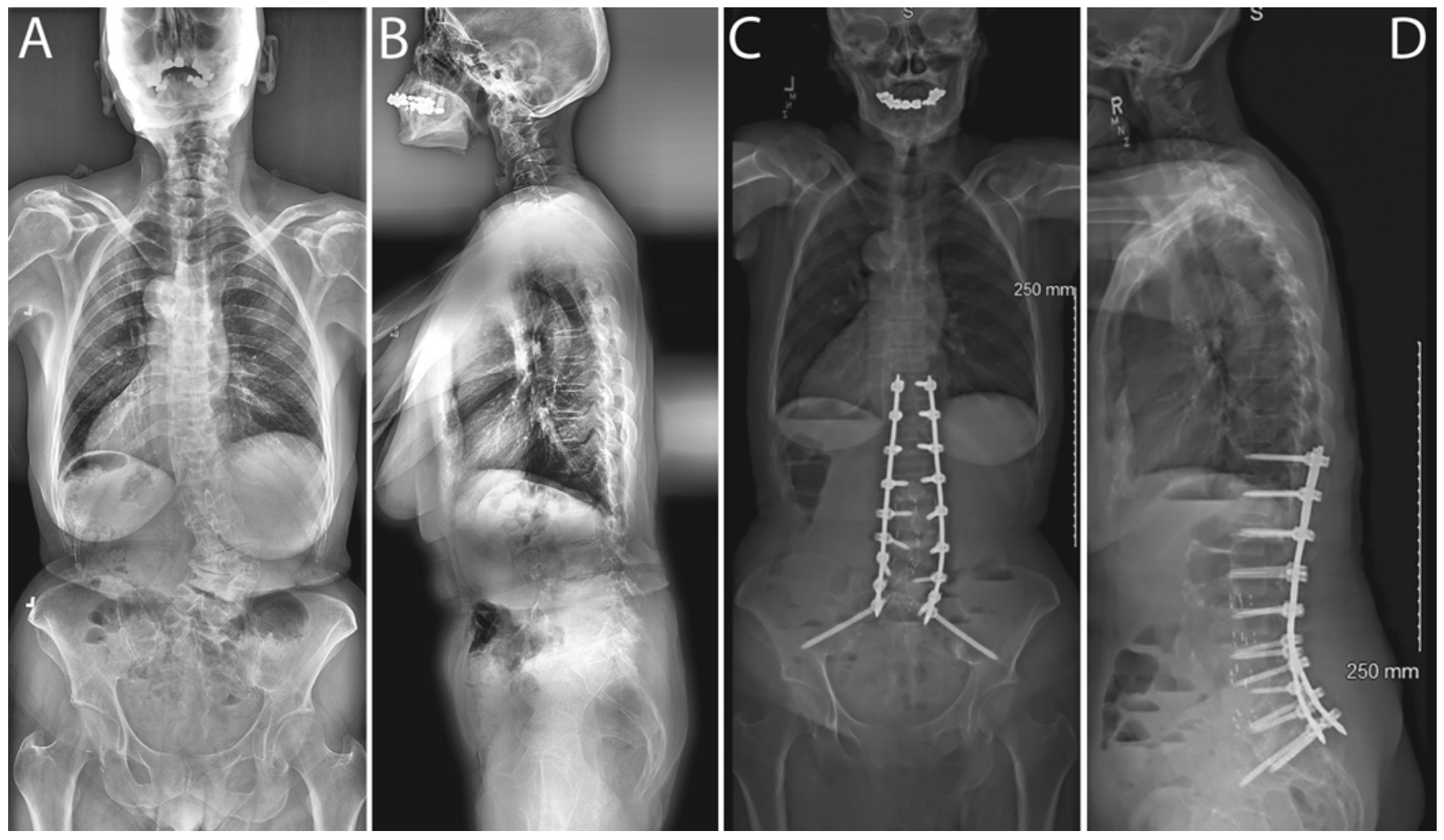

FIG. 2. Preoperative anteroposterior $(\mathbf{A})$ and lateral $(\mathbf{B})$ radiographs and postoperative anteroposterior $(\mathbf{C})$ and lateral (D) radiographs from a 74-year-old woman with kyphoscoliosis who underwent MIS surgery. Her preoperative values were: SVA 78.2 mm, TPA $23.4^{\circ}$, PT $22.1^{\circ}, \mathrm{PI}-\mathrm{LL} 28.7^{\circ}$, and CC $36.2^{\circ}$. 
TABLE 3. One-year outcomes in patients who underwent MIS surgery $(n=90)$

\begin{tabular}{lccc}
\hline \multicolumn{1}{c}{ Variable } & Baseline & 1-Yr Postop & p Value \\
\hline NRS back pain score & $7.0(2.0)$ & $2.9(2.6)$ & $<0.001$ \\
\hline NRS leg pain score & $5.7(2.9)$ & $2.5(2.9)$ & $<0.001$ \\
\hline ODI & $47.2(14.5)$ & $27.3(17.8)$ & $<0.001$ \\
\hline SRS-22 score $^{\circ}$ & $2.8(0.6)$ & $3.5(0.7)$ & $<0.001$ \\
\hline Sacral slope, $^{\circ}$ & $34.2(12.0)$ & $36.0(11.1)$ & 0.021 \\
\hline CC, $^{\circ}$ & $4.4(14.8)$ & $3.2(21.3)$ & 0.33 \\
\hline PT, $^{\circ}$ & $23.0(8.7)$ & $20.8(8.2)$ & 0.013 \\
\hline PI, $^{\circ}$ & $57.1(13.4)$ & $56.7(13.8)$ & 0.679 \\
\hline PI-LL, $^{\circ}$ & $14.9(16.5)$ & $6.9(11.3)$ & $<0.001$ \\
\hline LL, $^{\circ}$ & $42.3(19.0)$ & $49.8(14.5)$ & $<0.001$ \\
\hline SVA, ${ }^{\circ}$ mm & $60.3(58.6)$ & $39.8(43.6)$ & $<0.001$ \\
\hline TPA $^{\circ}$ & $21.7(10.0)$ & $18.1(8.2)$ & $<0.001$ \\
\hline
\end{tabular}

Data are presented as mean (SD).

was warranted in cases of severe deformity and the presumption that this could be best achieved with an open approach.

Of the variables studied, the need for an open decompression was the main driver for an open approach selection. Focal direct decompression can be performed with MIS surgical approaches, as evidenced by $33.1 \%$ of patients in the MIS group who had decompression. However, for certain deformity cases, it is generally not timeefficient to perform MIS direct decompression for diffuse symptomatic stenosis in the context of performing longersegment multilevel interbody fusions and instrumentation. Indirect decompression via LLIF has also been shown to be effective, ${ }^{12}$ but indirect decompression is less effective for certain types of stenosis. Wang et al. ${ }^{13}$ found that bone lateral recess stenosis was a risk factor for unsuccessful indirect decompression with LLIF. It is unclear what specific criteria were used by the participating surgeons to exclude the option of MIS direct and/or indirect decompression, but the necessity for open decompression was the most prominent differentiating selection factor.

Deformity surgery is associated with high risks of complications that are elevated in the elderly. Smith et al. ${ }^{14}$ analyzed 291 patients who underwent deformity surgery and noted a $78.9 \%$ complication rate in the 65 - to 86 -year-old age group versus a $55.4 \%$ complication rate in the 18- to 44-year-old age group. It is not surprising, therefore, that increasing age was associated with more likely selecting an MIS approach given the decreased exposure-related morbidity and perceived decreased complication risk.

Overall, both groups (open and MIS surgery) had significant improvements in both the clinical and radiographic parameters assessed. This suggests that appropriate selection was made in these cases, although it is not known whether the alternative approach could have resulted in a different, potentially better or worse outcome. The findings of this investigation reflect the perspective of the participating surgeons as a whole. One of the major drawbacks of such a statistical analysis of multicenter collective data is the potential to overgeneralize certain variables that con-
TABLE 4. Results of the multivariate analysis

\begin{tabular}{lcccr}
\hline \multicolumn{1}{c}{ Variable } & Reference & OR & $95 \% \mathrm{Cl}$ & p Value \\
\hline Age & MIS approach & 1.05 & $1.02-1.08$ & 0.002 \\
\hline Back pain & Open approach & 1.21 & $1.04-1.41$ & 0.016 \\
\hline CC & Open approach & 1.03 & $1.02-1.05$ & $<0.001$ \\
\hline Decompression & Open approach & 4.35 & $2.26-8.33$ & $<0.001$ \\
\hline TPA & Open approach & 1.09 & $1.01-1.18$ & 0.023 \\
\hline
\end{tabular}

tribute to surgical decision-making. It does not emphasize any individual surgeon's decision-making paradigm. For example, Anand et al. ${ }^{15,16}$ have shown an increased ability to treat spinal deformity patients with circumferential MIS techniques utilizing a specialized 2-stage protocol, even with severe spinopelvic malalignment.

A strength of this study is that the results showing that spinopelvic alignment, back pain, and need for decompression impact the decision to perform open surgery are likely a real-world representation of the adoption timeline of MIS surgical techniques for ASD. The adoption timeline to treat greater degrees of spinal deformity with MIS techniques may be well over 5 years. Increasing experience with the adoption of algorithmic protocols and advancing technology will probably further expand the selection of MIS techniques, even in severe ASD.

\section{Conclusions}

This investigation reviewed various factors influencing the choice of open versus minimally invasive techniques for ASD. Increasing age was the primary driver for selecting MIS surgery. Conversely, increasingly severe deformities and, in particular, the need for open decompression were the main factors influencing the decision for traditional open surgery. It is probable that with mounting experience, comfort with indirect decompression, refinement of treatment algorithms, and technological advancement, spine surgeons will increasingly employ MIS surgical techniques for ASD.

\section{Appendix}

The following centers participated in this study: Department of Neurosurgery, University of Michigan, Ann Arbor, Michigan; Department of Neurological Surgery, Oregon Health \& Science University, Portland, Oregon; Department of Neurosurgery, University of California, San Francisco, California; Department of Orthopaedic Surgery, Scripps Clinic, La Jolla, California; Spine Institute of Louisiana, Shreveport, Louisiana; Department of Neurosurgery, Barrow Neurological Institute, Phoenix, Arizona; Department of Neurosurgery, University of Miami, Florida; Department of Neurological Surgery, Rush University Medical Center, Chicago, Illinois; Department of Neurosurgery, University of Pittsburgh Medical Center, Pittsburgh,

Pennsylvania; and Department of Orthopaedics, Cedars-Sinai Medical Center, Los Angeles, California.

\section{References}

1. Anand N, Baron EM, Khandehroo B, Kahwaty S. Long-term 2- to 5-year clinical and functional outcomes of minimally 
invasive surgery for adult scoliosis. Spine (Phila Pa 1976). 2013;38(18):1566-1575.

2. Bae J, Lee SH. Minimally invasive spinal surgery for adult spinal deformity. Neurospine. 2018;15(1):18-24.

3. Park P, Wang MY, Lafage V, et al. Comparison of two minimally invasive surgery strategies to treat adult spinal deformity. J Neurosurg Spine. 2015;22(4):374-380.

4. Chou D, Mundis G, Wang M, et al. Minimally invasive surgery for mild-to-moderate adult spinal deformities: impact on intensive care unit and hospital stay. World Neurosurg. 2019; 127:e649-e655.

5. Lovecchio F, Qureshi SA. The current state of minimally invasive approaches to adult spinal deformity. Curr Rev Musculoskelet Med. 2019;16:318-327.

6. Ryan DJ, Protopsaltis TS, Ames CP, et al. T1 pelvic angle (TPA) effectively evaluates sagittal deformity and assesses radiographical surgical outcomes longitudinally. Spine (Phila Pa 1976). 2014;39(15):1203-1210.

7. Glassman SD, Berven S, Bridwell K, et al. Correlation of radiographic parameters and clinical symptoms in adult scoliosis. Spine (Phila Pa 1976). 2005;30(6):682-688.

8. Glassman SD, Bridwell K, Dimar JR, et al. The impact of positive sagittal balance in adult spinal deformity. Spine (Phila Pa 1976). 2005;30(18):2024-2029.

9. Lafage V, Schwab F, Patel A, et al. Pelvic tilt and truncal inclination: two key radiographic parameters in the setting of adults with spinal deformity. Spine (Phila Pa 1976). 2009;34(17):E599-E606.

10. Schwab F, Patel A, Ungar B, et al. Adult spinal deformitypostoperative standing imbalance: how much can you tolerate? An overview of key parameters in assessing alignment and planning corrective surgery. Spine (Phila Pa 1976). 2010;35(25):2224-2231.

11. Mummaneni PV, Park P, Shaffrey CI, et al. The MISDEF2 algorithm: an updated algorithm for patient selection in minimally invasive deformity surgery. J Neurosurg Spine. 2019;32(2):221-228.

12. Oliveira L, Marchi L, Coutinho E, Pimenta L. A radiographic assessment of the ability of the extreme lateral interbody fusion procedure to indirectly decompress the neural elements. Spine (Phila Pa 1976). 2010;35(26)(suppl):S331-S337.

13. Wang TY, Nayar G, Brown CR, et al. Bony lateral recess stenosis and other radiographic predictors of failed indirect decompression via extreme lateral interbody fusion: multiinstitutional analysis of 101 consecutive spinal levels. World Neurosurg. 2017;106:819-826.

14. Smith JS, Klineberg E, Lafage V, et al. Prospective multicenter assessment of perioperative and minimum 2-year postoperative complication rates associated with adult spinal deformity surgery. J Neurosurg Spine. 2016;25(1):1-14.

15. Anand N, Cohen JE, Cohen RB, et al. Comparison of a newer versus older protocol for circumferential minimally invasive surgical (CMIS) correction of adult spinal deformity (ASD) - evolution over a 10-year experience. Spine Deform. 2017;5(3):213-223.

16. Anand N, Kong C, Fessler RG. A staged protocol for circumferential minimally invasive surgical correction of adult spinal deformity. Neurosurgery. 2017;81(5):733-739.

\section{Disclosures}

Dr. Park reports being a consultant to Globus and NuVasive, receiving royalties from Globus, and receiving support of nonstudy-related clinical or research effort from DePuy and ISSG. Dr. Than reports being a consultant to Bioventus, and receiving honoraria from LifeNet Health and DJO. Dr. Mummaneni reports being a consultant to Stryker Spine, DePuy Synthes, and Globus; having direct stock ownership in Spinicity/ISD; receiving statistical analysis for study/writing or editorial assistance on the manuscript from ISSG; receiving royalties from DePuy Synthes, Thieme Publishers, and Springer Publishers; and receiving support of non-study-related clinical or research effort from $\mathrm{AO}$ Spine and NREF. Dr. Eastlack reports direct stock ownership in Seaspine, Alphatec, NuVasive, and Spine Innovation; being a consultant to NuVasive, Seaspine, SI Bone, Baxter, Stryker, and Medtronic; being a patent holder for NuTech, Globus, Seaspine, SI Bone, Spine Innovation, and Stryker; being on the Speakers Bureau for Radius; and receiving support of non-study-related clinical or research effort from Seaspine, NuVasive, Medtronic, and SI Bone. Dr. Uribe reports being a consultant to NuVasive. Dr. Wang reports being a consultant to DePuy-Synthes Spine, Medtronic, Stryker, Globus, and Spineology; being a patent holder for DePuy-Synthes Spine; and having direct stock ownership in ISD and Medical Device Partners. Dr. Okonkwo reports receiving royalties from NuVasive and Zimmer Biomet, and being a patent holder for Zimmer Biomet. Dr. Kanter reports receiving royalties from NuVasive and Zimmer Biomet. Dr. Anand reports being a consultant to Medtronic and DePuy Synthes; receiving royalties from Medtronic, Globus Medical, and Elsevier; being a patent holder for Medtronic; and having direct stock ownership in Globus Medical, Spinal Balance, and Spinal Simplicity. Dr. Chou reports being a consultant to Globus and Medtronic, and receiving royalties from Globus. Dr. Fu reports being a consultant to Globus, Johnson \& Johnson, SI Bone, and Atlas Spine. Dr. Shaffrey reports direct stock ownership in NuVasive; being a consultant to NuVasive, Medtronic, and SI Bone; receiving royalties from NuVasive and Medtronic; and being a patent holder for NuVasive, Medtronic, and Zimmer Biomet. Dr. Mundis reports being a consultant to NuVasive, Viseon, Seaspine, and Carlsmed; having direct stock ownership in NuVasive and Viseon; being a patent holder for $\mathrm{K} 2 \mathrm{M}$; and receiving royalties from NuVasive and $\mathrm{K} 2 \mathrm{M}$.

\section{Author Contributions}

Conception and design: Park, Than, Mummaneni, Eastlack, Fu, Mundis. Acquisition of data: all authors. Analysis and interpretation of data: Park, Mummaneni, Eastlack, Le, Mundis. Drafting the article: Park. Critically revising the article: Park, Than, Mummaneni, Eastlack, Uribe, Wang, Mundis. Reviewed submitted version of manuscript: all authors. Approved the final version of the manuscript on behalf of all authors: Park. Statistical analysis: Le.

\section{Correspondence}

Paul Park: University of Michigan, Ann Arbor, MI. ppark@med. umich.edu. 